TRANSACTIONS OF THE

AMERICAN MATHEMATICAL SOCIETY

Volume 356, Number 10, Pages 4075-4083

S 0002-9947(04)03557-3

Article electronically published on March 12, 2004

\title{
HOMOTOPY GROUPS OF $K$-CONTACT TORIC MANIFOLDS
}

\author{
EUGENE LERMAN
}

\begin{abstract}
Contact toric manifolds of Reeb type are a subclass of contact toric manifolds which have the property that they are classified by the images of the associated moment maps. We compute their first and second homotopy group terms of the images of the moment map. We also explain why they are $K$-contact.
\end{abstract}

\section{INTRODUCTION}

In this paper the first and second homotopy groups of certain toric symplectic cones or, equivalently, of certain contact toric manifolds are computed. The main result of the paper is Theorem 1.1 (the terms used in the statement are explained below).

Theorem 1.1. Let $G$ be a torus with Lie algebra $\mathfrak{g}$ and integral lattice $\mathbb{Z}_{G}=$ $\operatorname{ker}\{\exp : \mathfrak{g} \rightarrow G\}$. Let $(B, \xi=\operatorname{ker} \alpha)$ be a contact toric $G$-manifold of Reeb type with moment cone $C \subset \mathfrak{g}^{*}$, which is a strictly convex rational polyhedral cone. Let $\mathcal{L}$ denote the sublattice of $\mathbb{Z}_{G}$ generated by the normal vectors to the facets of $C$. The fundamental group of $B$ is the finite abelian group $\mathbb{Z}_{G} / \mathcal{L}$. The second homotopy group of $B$ is a free abelian group of $\operatorname{rank} N-\operatorname{dim} G$, where $N$ is the number of facets of the cone $C$.

Let us recall the necessary definitions (see $[\mathrm{L}$ for more details; see also [LS]). A manifold $B$ with a contact structure $\xi=\operatorname{ker} \alpha$ ( $\alpha$ is a contact form) is a toric $G$-manifold if there exists an effective action of a torus $G$ on $B$ preserving $\xi$ with $\operatorname{dim} B+1=2 \operatorname{dim} G$. By averaging over the group, if necessary, one can always assume that the torus $G$ preserves a contact form $\alpha$ defining $\xi$.

Given an action of a Lie group $G$ on a manifold $B$ preserving a contact form $\alpha$, the corresponding $\alpha$-moment map $\Psi_{\alpha}: B \rightarrow \mathfrak{g}^{*}\left(\mathfrak{g}^{*}\right.$ denotes the vector space dual of the Lie algebra $\mathfrak{g}$ of $G$ ) is defined by

$$
\left\langle\Psi_{\alpha}(b), X\right\rangle=\alpha_{b}\left(X_{B}(b)\right)
$$

for all $b \in B$, and all $X \in \mathfrak{g}$. As usual $\langle\cdot, \cdot\rangle$ denotes the canonical pairing between $\mathfrak{g}^{*}$ and $\mathfrak{g}$, and $X_{B}$ denotes the vector field on $B$ induced by $X$. If $f \in C^{\infty}(B)^{G}$ is an invariant function, then $\alpha^{\prime}=e^{f} \alpha$ is another contact form defining the same contact distribution $\xi$ as $\alpha$. Clearly $\Psi_{e^{f} \alpha}=e^{f} \Psi_{\alpha}$, so the moment map is an invariant of

Received by the editors December 23, 2002 and, in revised form, July 11, 2003.

2000 Mathematics Subject Classification. Primary 53D10; Secondary 53D20.

The author was supported by NSF grant DMS-980305. 
the contact form and not of the contact distribution. On the other hand the subset $C(\Psi)=C\left(\Psi_{\alpha}\right)$ of $\mathfrak{g}^{*}$ for an $\alpha$-moment map $\Psi_{\alpha}: B \rightarrow \mathfrak{g}^{*}$ defined by

$$
C(\Psi)=\left\{t \Psi_{\alpha}(b) \mid t \geq 0, b \in B\right\}
$$

depends only on the action of $G$ on $B$ and on the contact distribution $\xi$ but not on the contact form $\alpha$ per se. We will refer to $C(\Psi)$ as the moment cone of the action.

Since a moment map $\Psi_{\alpha}: B \rightarrow \mathfrak{g}^{*}$ completely encodes the action of $G$ on $(B, \alpha)$ we regard a contact toric $G$-manifold as a triple $\left(B, \xi=\operatorname{ker} \alpha, \Psi_{\alpha}: B \rightarrow \mathfrak{g}^{*}\right)$. Note that the symplectization $(M, \omega):=\left(B \times \mathbb{R}, d\left(e^{t} \alpha\right)\right)(t \in \mathbb{R})$ is a noncompact symplectic toric manifold with moment map $\Phi(b, t)=e^{t} \Psi_{\alpha}(b)$. Remark that $\Phi(M) \cup\{0\}=C(\Psi)$. Conversely, if a symplectic toric $G$-manifold $\left(M, \omega, \Phi: M \rightarrow \mathfrak{g}^{*}\right)$ is a symplectic cone, i.e., if there is a free proper action $\left\{\rho_{t}\right\}$ of $\mathbb{R}$ on $M$ commuting with the action of $G$ such that $\rho_{t}^{*} \omega=e^{t} \omega$, then $M / \mathbb{R}$ is naturally a contact toric manifold.

A contact manifold $(B, \xi=\operatorname{ker} \alpha)$ with an action of a torus $G$ preserving $\alpha$ is of Reeb type if there is $X \in \mathfrak{g}$ such that the function $\left\langle\Psi_{\alpha}, X\right\rangle=\iota\left(X_{B}\right) \alpha$ is strictly positive. By a result of Boyer and Galicki [BG] (see also Theorem 4.3 in $[\mathrm{LS}]$ ), the moment cone of a contact toric $G$ manifold of Reeb type is a strictly convex rational polyhedral cone. "Strictly convex" means that the moment cone contains no linear subspaces of positive dimension, i.e., it is a cone on a polytope. "Rational polyhedral" means that there exist vectors $\mu_{1}, \ldots, \mu_{N}$ in the integral lattice $\mathbb{Z}_{G}:=\operatorname{ker}(\exp : \mathfrak{g} \rightarrow G)$ of the torus $G$ such that

$$
C(\Psi)=\left\{\eta \in \mathfrak{g}^{*} \mid\left\langle\eta, \mu_{j}\right\rangle \geq 0, j=1, \ldots, N\right\} .
$$

There are several reasons for wanting to compute the homotopy groups of contact toric manifolds of Reeb type.

1. All contact manifolds of Reeb type are $K$-contact (see Proposition 3.1 below), hence the title of the paper. In fact contact toric manifolds of Reeb type are Sasakian, as proved by Boyer and Galicki (Theorem 5.3 in [BG]). Methods recently developed by Boyer, Galicki, Mann and others use Sasakian structures to obtain explicit positive Einstein metrics.

Conversely, as was pointed out by the referee, any contact toric manifold with an invariant $K$-contact structure is of Reeb type (Proposition 3.2 below).

2. A classification of contact toric manifolds $[\mathrm{L}$ shows that they naturally fall into two classes: Reeb type and the rest. The manifolds of Reeb type are classified by their moment map images, i.e., strictly convex rational polyhedral cones. This is the class of contact toric manifolds most analogous to the symplectic toric manifolds classified by Delzant [D].

The rest of contact toric manifolds either are not classified by their moment cones or the corresponding moment cones are not strictly convex. On the other hand, topologically they are easy to understand: they are either $S^{2} \times S^{1}$, or products $\mathbb{T}^{k} \times S^{k+2 l-1}(k>1, l \geq 0)$ or principal $\mathbb{T}^{3}$ bundles over $S^{2}$. Note that for the latter the moment cones are all of $\mathfrak{g}^{*}$ and, in particular, give us no information as to which principal $\mathbb{T}^{3}$ bundle we are dealing with. Thus, for contact toric manifolds that are not of Reeb type there is no natural analogue of Theorem 1.1. On the other hand, such a theorem is unnecessary.

3. One motivation for studying the topology of contact toric manifolds is their apparent difference from (topological) toric manifolds. Recall that in 1991 Davis 
and Januszkiewiecz defined (topological) toric manifolds as manifolds with torus action locally modeled on the standard action of $\mathbb{T}^{n}$ on $\mathbb{C}^{n}$ and having a simple polytope as the orbit space [DJ]. Such a manifold is determined by a polytope and a characteristic function, a function that assigns a 1-parameter subgroup of the torus to every facet of the polytope. They proved a beautiful formula for the integral cohomology ring of a toric manifold; it is the Stanley-Reisner ring of the polytope modulo an ideal determined by the characteristic function (for smooth projective toric varieties the formula is known as the Danilov-Jurkiewicz theorem). In particular the cohomology ring is generated by elements of degree two, odd dimensional cohomology vanishes and there is no torsion. They also proved that such manifolds are simply connected. In contrast, the odd dimension cohomology of a contact toric manifold need not vanish $\left(\mathrm{cf} . \mathbb{R} P^{3}\right)$, there is torsion and the fundamental group need not be trivial.

4. Another motivation comes from the study of completely integrable geodesic flows. According to Toth and Zelditch [TZ, a geodesic flow on a manifold $Q$ is toric integrable if there exists a homogeneous completely integrable action of a torus on the punctured cotangent bundle $T^{*} Q \backslash Q$ which preserves the geodesic flow. Naturally in this case the co-sphere bundle $S\left(T^{*} Q\right)$ is a contact toric manifold. It would be interesting to find a topological obstruction to the existence of a toric integrable geodesic flow on a compact manifold $Q$, and for that one needs to understand the topology of contact toric manifolds.

We now outline the proof of Theorem 1.1.

1) Since a contact manifold $B$ is homotopy equivalent to its symplectization $M=B \times \mathbb{R}$, we compute the homotopy groups of the symplectization.

2) The symplectization $M$ of $B$ is the symplectic quotient at 0 of $\mathbb{C}^{N} \backslash\{0\}$ by a compact abelian group $T$ with $\pi_{0}(T)=\mathbb{Z}_{G} / \mathcal{L}$ and $\operatorname{dim} T=N-\operatorname{dim} G$. That is to say, $M=\left(\Phi_{T}^{-1}(0) \backslash\{0\}\right) / T$, where $\Phi_{T}: \mathbb{C}^{N} \rightarrow \mathfrak{t}^{*}$ denotes the $T$-moment map for the linear action of $T$ on $\mathbb{C}^{N}$.

3) The set $\Phi_{T}^{-1}(0) \backslash\{0\}$ has the homotopy type of $\mathbb{C}^{N} \backslash\left(V_{1} \cup V_{2} \cup \ldots \cup V_{r}\right)$, where each $V_{j} \subset \mathbb{C}^{N}$ is a linear subspace of complex codimension at least 2 . Hence $\pi_{0}\left(\Phi_{T}^{-1}(0) \backslash\{0\}\right)=\pi_{1}\left(\Phi_{T}^{-1}(0) \backslash\{0\}\right)=\pi_{2}\left(\Phi_{T}^{-1}(0) \backslash\{0\}\right)=*$.

4) Since the group $T$ acts freely on $\Phi_{T}^{-1}(0) \backslash\{0\}$, we see from the long exact sequence of homotopy groups for the fibration $T \rightarrow\left(\Phi_{T}^{-1}(0) \backslash\{0\}\right) \rightarrow M$ that

$$
\pi_{1}(M)=\pi_{0}(T) \text { and } \quad \pi_{2}(M)=\pi_{1}(T) .
$$

The details of the argument are the subject of the next section. In the last section we explain the connection between torus actions of Reeb type and being $K$-contact.

A note on notation. Throughout the paper the Lie algebra of a Lie group denoted by a capital Roman letter will be denoted by the same small letter in the fraktur font: thus $\mathfrak{g}$ denotes the Lie algebra of a Lie group $G$, etc. The natural pairing between $\mathfrak{g}$ and its vector space dual $\mathfrak{g}^{*}$ is denoted by $\langle\cdot, \cdot\rangle$. If $A: V \rightarrow W$ is a linear map, we denote the corresponding map on the dual spaces by $A^{*}, A^{*}: W^{*} \rightarrow V^{*}$.

When a Lie group $G$ acts on a manifold $M$ we denote the action by an element $g \in G$ on a point $x \in M$ by $g \cdot x, G \cdot x$ denotes the $G$-orbit of $x$, and so on. The vector field induced on $M$ by an element $X$ of the Lie algebra $\mathfrak{g}$ of $G$ is denoted by $X_{M}$. Thus $X_{M}(m)=\left.\frac{d}{d t}\right|_{t=0}(\exp t X) \cdot m$. 
For us a torus is a compact connected abelian group. If $G$ is a torus, we denote its weight lattice by $\mathbb{Z}_{G}^{*}$; it is a subgroup of $\mathfrak{g}^{*}$. The dual lattice of $\mathbb{Z}_{G}^{*}$ is the integral lattice $\mathbb{Z}_{G}$. Recall that $\mathbb{Z}_{G}=\operatorname{ker}(\exp : \mathfrak{g} \rightarrow G)$. Thus $G=\mathfrak{g} / \mathbb{Z}_{G}$.

\section{Proof of the main Result, Theorem 1.1}

It was proved in $[\mathrm{L}]$ that the moment cone $C(\Psi)$ of a (compact connected) contact toric $G$-manifold $\left(B, \xi=\operatorname{ker} \alpha, \Psi_{\alpha}: B \rightarrow \mathfrak{g}^{*}\right)$ of Reeb type is a good cone. This means the following. Let $\left\{F_{i}\right\}$ denote the set of facets (codimension one faces) of $C(\Psi)$. Since $C(\Psi)$ is rational, each facet is of the form

$$
F_{i}=\left\{\eta \in C(\Psi) \mid\left\langle\eta, \mu_{i}\right\rangle=0\right\}
$$

for some primitive vector $\mu_{i}$ in the integral lattice $\mathbb{Z}_{G}$ of $G$. Then

(1) every codimension $\ell, 0<\ell<\operatorname{dim} G$, face $F$ of $C(\Psi)$ can be written uniquely as

$$
F=F_{i_{1}} \cap \ldots \cap F_{i_{\ell}}
$$

where $F_{i_{j}}$ 's are the facets containing $F$, and

(2) the $\mathbb{Z}$-module generated by the normals to the facets $F_{i_{1}}, \ldots, F_{i_{\ell}}$ is a direct summand of $\mathbb{Z}_{G}$ of rank $\ell$.

We have a uniqueness result $\left[\mathrm{L}\right.$ : if $\left(B, \xi=\operatorname{ker} \alpha, \Psi_{\alpha}\right)$ and $\left(B^{\prime}, \xi^{\prime}=\operatorname{ker} \alpha^{\prime}, \Psi_{\alpha^{\prime}}\right)$ are two (compact connected) contact toric manifolds of Reeb type and the moment cones are equal, then the contact toric manifolds are equivariantly contactomorphic.

There is also a corresponding existence result. Given a good polyhedral cone $C \subset \mathfrak{g}^{*}$ (where $\mathfrak{g}^{*}$ is the dual of the Lie algebra of a torus $G$ ), there exists a compact connected contact toric $G$-manifold $\left(B_{C}, \xi_{C}=\operatorname{ker} \alpha_{C}, \Psi_{\alpha_{C}}\right)$ with the moment cone $C\left(\Psi_{\alpha_{C}}\right)$ equal to $C$ (Theorem 2.18(4) of $\left.[\mathrm{L}]\right)$. Moreover $\left(B_{C}, \xi_{C}=\operatorname{ker} \alpha_{C}, \Psi_{\alpha_{C}}\right)$ can be constructed as a contact quotient of the standard odd dimensional sphere. In fact it is more convenient to construct the symplectization $\left(M_{C}, \omega_{C}, \Phi_{C}: M_{C} \rightarrow \mathfrak{g}^{*}\right)$ of $\left(B_{C}, \alpha_{C}, \Psi_{\alpha_{C}}: B_{C} \rightarrow \mathfrak{g}^{*}\right)$. Then for any contact toric $G$-manifold $\left(B^{\prime}, \xi^{\prime}=\right.$ ker $\left.\alpha^{\prime}, \Psi_{\alpha^{\prime}}\right)$ with $C\left(\Psi_{\alpha^{\prime}}\right)=C$, we have

$$
\pi_{1}\left(M_{C}\right)=\pi_{1}\left(B^{\prime}\right), \quad \pi_{2}\left(M_{C}\right)=\pi_{2}\left(B^{\prime}\right)
$$

and so on. Note that the moment map image $\Phi_{C}\left(M_{C}\right)$ is $C \backslash\{0\}$.

Recall from $[\mathrm{L}]$ the construction of the symplectic toric manifold $\left(M_{C}, \omega_{C}, \Phi_{C}\right.$ : $\left.M_{C} \rightarrow \mathfrak{g}^{*}\right)$. As above let $\mu_{1}, \ldots, \mu_{N} \in \mathbb{Z}_{G}$ denote the primitive inward normals to the facets of the good strictly convex cone $C$. Since $C$ is strictly convex and has nonempty interior, $\operatorname{span}_{\mathbb{R}}\left\{\mu_{i}\right\}=\mathfrak{g}$. Hence the abelian group $\mathbb{Z}_{G} / \mathcal{L}$, where $\mathcal{L}=\operatorname{span}_{\mathbb{Z}}\left\{\mu_{i}\right\}$, is finite. Consider the $\mathbb{Z}$-linear map

$$
\varpi: \mathbb{Z}^{N} \rightarrow \mathbb{Z}_{G}, \quad \varpi\left(a_{1}, \ldots, a_{N}\right)=\sum a_{i} \mu_{i} .
$$

Its cokernel is $\mathbb{Z}_{G} / \mathcal{L}$. It extends to a surjective $\mathbb{R}$-linear map

$$
\tilde{\varpi}: \mathbb{R}^{N} \rightarrow \mathfrak{g}, \quad \tilde{\varpi}\left(a_{1}, \ldots, a_{N}\right)=\sum a_{i} \mu_{i},
$$

which drops down to a surjective Lie group homomorphism

$$
\begin{aligned}
& \bar{\varpi}: \mathbb{T}^{N}=\mathbb{R}^{N} / \mathbb{Z}^{N} \rightarrow \mathfrak{g} / \mathbb{Z}_{G}=G, \\
& \bar{\varpi}\left(\left[a_{1}, \ldots, a_{N}\right]\right)=\exp \left(\tilde{\varpi}\left(a_{1}, \ldots, a_{N}\right)\right)=\exp \left(\sum a_{i} \mu_{i}\right) .
\end{aligned}
$$

Here $\left[a_{1}, \ldots, a_{N}\right]$ denotes the class of $\left(a_{1}, \ldots, a_{N}\right) \in \mathbb{R}^{N}$ in $\mathbb{T}^{N}$ and $\exp : \mathfrak{g} \rightarrow G$ denotes the exponential map. Let $T=\operatorname{ker} \overline{\bar{\omega}}$; it is a closed but not necessarily 
connected subgroup of $\mathbb{T}^{N}$. The standard linear action of $\mathbb{T}^{N}$ on $\mathbb{C}^{N}$ preserving the standard symplectic form $\sqrt{-1} \sum d z_{j} \wedge d \bar{z}_{j}$ gives rise to a linear symplectic action of $T \subset \mathbb{T}^{N}$. Denote the corresponding homogeneous moment map by $\Phi_{T}$; $\Phi_{T}: \mathbb{C}^{N} \rightarrow \mathfrak{t}^{*}$. The moment map $\Phi: \mathbb{C}^{N} \rightarrow\left(\mathbb{R}^{N}\right)^{*}$ for the standard action of $\mathbb{T}^{N}$ on $\mathbb{C}^{N}$ is given by the formula

$$
\Phi\left(z_{1}, \ldots, z_{N}\right)=\sum\left|z_{j}\right|^{2} e_{j}^{*}
$$

where $e_{1}^{*}, \ldots, e_{N}^{*}$ is the standard basis of $\left(\mathbb{R}^{N}\right)^{*}$. Hence, if $\iota: \mathfrak{t} \rightarrow \mathbb{R}^{N}$ denotes the inclusion of the Lie algebra $\mathfrak{t}$ of $T$, we have $\Phi_{T}=\iota^{*} \circ \Phi$. We recall from [L]:

Lemma 2.1. We use the notation above. The set $\Phi_{T}^{-1}(0) \backslash\{0\}$ is a manifold. The group $T$ acts freely on this manifold. The symplectic manifold

$$
M:=\left(\Phi_{T}^{-1}(0) \backslash\{0\}\right) / T
$$

is the desired $G=\mathbb{T}^{N} / T$ symplectic manifold, that is, it is a symplectic cone and the image of the $G$-moment map is $C \backslash\{0\}$. In particular $\Phi\left(\Phi_{T}^{-1}(0)\right)=\tilde{\varpi}^{*}(C)$, where $\tilde{\varpi}^{*}: \mathfrak{g}^{*} \rightarrow\left(\mathbb{R}^{N}\right)^{*}$ is dual to $\tilde{\varpi}(c f .(2.2))$.

Our proof of Theorem 1.1 is based on two lemmas. The first one describes the group $\pi_{0}(T)$ of connected components of $T$ :

Lemma 2.2. Let $T \subset \mathbb{T}^{N}$ be as above. Then $\pi_{0}(T)=\mathbb{Z}_{G} / \mathcal{L}$, where, as above, $\mathcal{L}$ is the sublattice of the integral lattice $\mathbb{Z}_{G}$ spanned by the primitive normals to the facets of the cone $C$.

The second lemma shows that the manifold $\Phi_{T}^{-1}(0) \backslash\{0\}$ has the homotopy type of $\mathbb{C}^{N} \backslash\left(V_{1} \cup \ldots \cup V_{r}\right)$, where $V_{j} \subset \mathbb{C}^{N}$ are complex linear subspaces of complex codimension at least 2 . In fact the subspaces $V_{j}$ being deleted are determined by the combinatorics of the polyhedral cone $C$. To make this precise we need a few definitions.

For a subset $I \subset\{1, \ldots, N\}$ define the corresponding coordinate subspace $V_{I}$ by

$$
V_{I}:=\left\{z \in \mathbb{C}^{N} \mid j \in I \Rightarrow z_{j}=0\right\}=\bigcap_{j \in I}\left\{z_{j}=0\right\} .
$$

For each $j \in\{1, \ldots, N\}$ the $j$ th facet $F_{j}$ of the cone $C$ satisfies

$$
F_{j}=C \cap\left\{\eta \in \mathfrak{g}^{*} \mid\left\langle\eta, \mu_{j}\right\rangle=0\right\} .
$$

Now consider the set

$$
\mathcal{U}:=\left\{I \subset\{1, \ldots, N\} \mid \bigcap_{j \in I} F_{j}=\{0\}\right\},
$$

the collection of subsets $I$ of $\{1, \ldots, N\}$ such that the facets indexed by the elements of $I$ intersect only at the vertex.

Lemma 2.3. The manifold $\Phi_{T}^{-1}(0) \backslash\{0\}$ has the same homotopy type as

$$
\mathbb{C}^{N} \backslash \bigcup_{I \in \mathcal{U}} V_{I}
$$

Let us assume the lemmas for a moment and prove the main theorem, Theorem 1.1. 
Proof of Theorem [1.1] As was remarked previously, it is enough to prove that the symplectic toric manifold $M_{C}=M=\left(\Phi_{T}^{-1}(0) \backslash\{0\}\right) / T$ has the properties that $\pi_{1}(M)=\mathbb{Z}_{G} / \mathcal{L}$ and that $\pi_{2}\left(M_{C}\right)=\mathbb{Z}^{d}$, where $d=N-\operatorname{dim} G$ and $N$, as before, denotes the number of the facets of the cone $C$. Since $T$ acts freely on $Z:=$ $\Phi_{T}^{-1}(0) \backslash\{0\}$, we have a long exact sequence of homotopy groups

$$
\cdots \rightarrow \pi_{2}(Z) \rightarrow \pi_{2}(M) \rightarrow \pi_{1}(T) \rightarrow \pi_{1}(Z) \rightarrow \pi_{1}(M) \rightarrow \pi_{0}(T) \rightarrow \pi_{0}(Z) \rightarrow \pi_{0}(M) .
$$

Since every facet $F_{j}$ of $C$ is not $\{0\}$, the set $\mathcal{U}$ contains no singletons. Since $\operatorname{dim}_{\mathbb{C}} V_{I}=N-|I|$, it follows that for any $I \in \mathcal{U}, \operatorname{codim}_{\mathbb{C}} V_{I}=|I| \geq 2$. Hence by Lemma $2.3 Z$ is connected and the homotopy groups $\pi_{2}(Z), \pi_{1}(Z)$ are trivial. It follows from (2.5) that

$$
\pi_{2}(M)=\pi_{1}(T) \text { and } \pi_{1}(M)=\pi_{0}(T) .
$$

By Lemma $2.2 \pi_{0}(T)=\mathbb{Z}_{G} / \mathcal{L}$. Clearly $\pi_{1}(T)=\mathbb{Z}^{d}, d=\operatorname{dim} T=\operatorname{dim} \mathbb{T}^{N}-$ $\operatorname{dim} G$.

Proof of Lemma 2.2. This is a simple application of the Snake lemma. Consider the commuting diagram

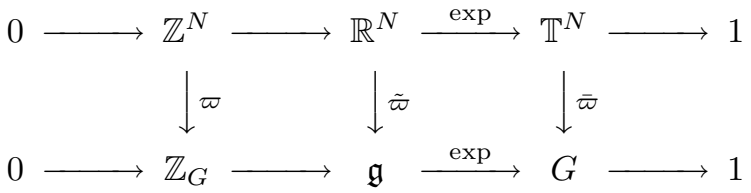

By the Snake lemma we have a long exact sequence

$$
\text { ker } \varpi \rightarrow \operatorname{ker} \tilde{\varpi} \rightarrow \operatorname{ker} \bar{\varpi} \rightarrow \operatorname{coker} \varpi \rightarrow \operatorname{coker} \tilde{\varpi} \rightarrow \operatorname{coker} \bar{\varpi} \text {. }
$$

By construction $\tilde{\varpi}$ is onto, hence coker $\tilde{\varpi}=0$. On the other hand coker $\varpi=$ $\mathbb{Z}_{G} / \mathcal{L}$. By definition ker $\bar{\varpi}=T$, ker $\tilde{\varpi}=\mathfrak{t}$ and the map ker $\tilde{\varpi} \rightarrow$ ker $\bar{\varpi}$ is simply the exponential map exp $: \mathfrak{t} \rightarrow T$. Since coker $(\exp : \mathfrak{t} \rightarrow T)$ is $\pi_{0}(T)$, we get $\pi_{0}(T) \simeq \mathbb{Z}_{G} / \mathcal{L}$.

Proof of Lemma[2.3. We keep the notation of the discussion above. The proof is an elementary application of the correspondence between symplectic quotients and Geometric Invariant Theory (GIT) quotients as developed by Mumford, Guillemin, Sternberg, Kirwan, Neeman, Sjamaar and others. The key point is that the GIT quotient $\mathbb{C}^{N} / / T^{\mathbb{C}}$ and the symplectic quotient $\Phi_{T}^{-1}(0) / T$ are isomorphic as stratified spaces. It will be most convenient for us to quote $[\underline{S}$, where Kirwan's results on the isomorphism between symplectic and GIT quotients were suitably refined.

(1) By Lemma 2.1 the group $T$ acts freely on the manifold $Z=\Phi_{T}^{-1}(0) \backslash\{0\}$.

(2) By Example 2.3 of $[\underline{\mathrm{S}}], \Phi_{T}$ is admissible in the sense of $[\underline{\mathrm{S}}]$, p. 109, and the set of analytically semistable points $\left(\mathbb{C}^{N}\right)^{s s}$ for the action of $T$ on $\mathbb{C}^{N}$ is all of $\mathbb{C}^{N}$.

(3) By Proposition 1.6 of $\left[\underline{\mathrm{S}}\right.$, for any point $z \in \mathbb{C}^{N}$ the stabilizer in the complexified group is the complexification of the stabilizer:

$$
\left(T^{\mathbb{C}}\right)_{z}=\left(T_{z}\right)^{\mathbb{C}}
$$

Hence by $(1),\left(T^{\mathbb{C}}\right)_{z}$ is trivial for all $z \in Z$.

(4) By Proposition 2.4(ii) of $\left[\underline{S}\right.$ the orbit $T^{\mathbb{C}} \cdot z$ is closed in $\left(\mathbb{C}^{N}\right)^{s s}=\mathbb{C}^{N}$ if and only if $T^{\mathbb{C}} \cdot z \cap \Phi_{T}^{-1}(0) \neq \emptyset$. Thus

$$
\left\{z \in \mathbb{C}^{N} \mid T^{\mathbb{C}} \cdot z \text { is closed }\right\}=\{0\} \cup T^{\mathbb{C}} \cdot Z .
$$


(5) Since the actions of $\left(\mathbb{T}^{N}\right)^{\mathbb{C}}$ and $T^{\mathbb{C}}$ commute, the union (2.6) of closed $T^{C}$ orbits is $\left(\mathbb{T}^{N}\right)^{\mathbb{C}}$ invariant. Hence, since $\{0\}$ is fixed by $\left(\mathbb{T}^{N}\right)^{\mathbb{C}}$, the set

$$
S:=T^{\mathbb{C}} \cdot Z
$$

is $\left(\mathbb{T}^{N}\right)^{\mathbb{C}}$ invariant.

(6) Proposition 2.4(iii) of $[\mathrm{S}]$ implies that $\left(T^{\mathbb{C}} \cdot Z\right) / T^{\mathbb{C}}=Z / T$. Combining this with (3) we see that $S$ is a $T^{\mathbb{C}} / T$-bundle over $Z$. Since $T^{\mathbb{C}} / T$ is diffeomorphic to the Lie algebra $\mathfrak{t}$ of $T$, the manifolds $S$ and $Z$ are homotopy equivalent.

(7) For any subset $I$ of $\{1, \ldots, N\}$ define

$$
\stackrel{\circ}{V}_{I}=\left\{z \in V_{I} \mid z_{j} \neq 0 \text { for } j \notin I\right\},
$$

the "interior" of the coordinate subspace $V_{I}$. The set $\stackrel{\circ}{V}_{I}$ is a single $\left(\mathbb{T}^{N}\right)^{\mathbb{C}}$ orbit. It satisfies

$$
\stackrel{\circ}{V}_{I}=V_{I} \backslash \bigcup_{I^{\prime} \supset I, I^{\prime} \neq I} V_{I^{\prime}}
$$

We claim that

$$
\stackrel{\circ}{V}_{I} \subset S \Leftrightarrow \bigcap_{j \in I} F_{j} \text { is a nonzero face of } C \text {. }
$$

Proof of (2.7). Note that since $S$ is $\left(\mathbb{T}^{N}\right)^{\mathbb{C}}$ invariant and $\stackrel{\circ}{V}_{I}$ is a $\left(\mathbb{T}^{N}\right)^{\mathbb{C}}$ orbit, $\stackrel{\circ}{V}_{I} \subset S \Leftrightarrow \stackrel{\circ}{V}_{I} \cap S \neq \emptyset$. Also, since $z \in S \Leftrightarrow T^{\mathbb{C}} \cdot z \cap Z \neq \emptyset$ and since $S$ is $\left(\mathbb{T}^{N}\right)^{\mathbb{C}}$ invariant, we have

$$
z \in S \Leftrightarrow\left(\mathbb{T}^{N}\right)^{\mathbb{C}} \cdot z \cap Z \neq \emptyset .
$$

As before let $\mu_{j} \in \mathbb{Z}_{G}$ denote the (primitive inward pointing) normal to the facet $F_{j}$ of $C$. Suppose $F_{I}:=\bigcap_{j \in I} F_{j}$ is a nonzero face of $C$. Pick a point $\eta$ in the relative interior of $F_{I}$. Then $\left\langle\eta, \mu_{k}\right\rangle>0$ for all $k \notin I$. Let $z_{j}^{\eta}=\sqrt{\left\langle\eta, \mu_{j}\right\rangle} ; z^{\eta}:=\left(z_{1}^{\eta}, \ldots, z_{N}^{\eta}\right)$ satisfies

$$
\left\langle\Phi\left(z^{\eta}\right), e_{j}\right\rangle=\left|z_{j}^{\eta}\right|^{2}=\left\langle\eta, \mu_{j}\right\rangle=\left\langle\eta, \tilde{\varpi}\left(e_{j}\right)\right\rangle=\left\langle\tilde{\varpi}^{*}(\eta), e_{j}\right\rangle
$$

for all $j$, where, as before, $e_{1}, \ldots, e_{N}$ is the standard basis of $\mathbb{R}^{N}, \Phi: \mathbb{C}^{N} \rightarrow$ $\left(\mathbb{R}^{N}\right)^{*}$ is the moment map for the standard action of $\mathbb{T}^{N}$ on $\mathbb{C}^{N}$ (see (2.3)) and $\tilde{\varpi}: \mathbb{R}^{N} \rightarrow \mathfrak{g}$ is the surjective map defined earlier by (2.2). Hence $\Phi\left(z^{\eta}\right)=\tilde{\varpi}^{*}(\eta)$, so $z^{\eta} \in \Phi^{-1}\left(\tilde{\varpi}^{*}(e t a)\right)$. Since $\eta \neq 0$ we have

$$
\emptyset \neq T^{\mathbb{C}} \cdot z^{\eta} \cap\left(\Phi^{-1}\left(\tilde{\varpi}^{*}(C)\right) \backslash\{0\}\right)=T^{\mathbb{C}} \cdot z^{\eta} \cap Z,
$$

where we used the fact that $\Phi^{-1}\left(\tilde{\varpi}^{*}(C)\right)=Z \cup\{0\}$. Also $z^{\eta} \in \stackrel{\circ}{V}_{I}$ since $\left|z_{j}^{\eta}\right|^{2}=$ $\left\langle\eta, \mu_{j}\right\rangle$ for all $j$ and $\left\langle\eta, \mu_{j}\right\rangle>0$ for $j \notin I$. This proves that if the intersections $\bigcap_{j \in I} F_{j}$ is a nonzero face of $C$, then $\stackrel{\circ}{V}_{I} \cap Z \neq \emptyset$. Hence $\stackrel{\circ}{V}_{I} \cap S \neq \emptyset$ and therefore $\stackrel{\circ}{V}_{I} \subset S$.

Conversely, suppose $\stackrel{\circ}{V}_{I} \subset S$. Then $\stackrel{\circ}{V}_{I} \cap Z \neq \emptyset$. For any $z \in \stackrel{\circ}{V}_{I} \cap Z$ we have: $\Phi(z) \in \tilde{\varpi}^{*}(C),\left|z_{j}\right|^{2} \neq 0$ for $j \notin I$, and $\left|z_{j}\right|^{2}=0$ for $j \in I$. Therefore $\Phi(z)=\tilde{\varpi}^{*}(\eta)$ for some $\eta \in C$ and $\left\langle\eta, \mu_{j}\right\rangle \neq 0$ for all $j \notin I,\left\langle\eta, \mu_{j}\right\rangle=0$ for all $j \in I$. Hence

$$
\eta \in\left(\bigcap_{j \notin I}\left\{\eta \in \mathfrak{g}^{*} \mid\left\langle\eta, \mu_{j}\right\rangle>0\right\}\right) \cap\left(\bigcap_{j \in I}\left\{\eta \in \mathfrak{g}^{*} \mid\left\langle\eta, \mu_{j}\right\rangle=0\right\}\right) .
$$

Thus $F_{I}=\bigcap_{j \in I} F_{j}$ is a nonzero face of $C$. This proves (2.7). 
(8) If $\bigcap_{j \in I} F_{j}=\{0\}$, then for any $I^{\prime} \supset I, \bigcap_{j \in I^{\prime}} F_{j}=\{0\}$ as well. Since $V_{I}=\bigcup_{I^{\prime} \supseteq I} \stackrel{\circ}{V}_{I^{\prime}}$, (2.7) implies that

$$
S=\mathbb{C}^{N} \backslash \bigcup_{I \in \mathcal{U}} V_{I} .
$$

By (6) $Z=\Phi_{T}^{-1}(0) \backslash\{0\}$ is homotopy equivalent to $S=\mathbb{C}^{N} \backslash \bigcup_{I \in \mathcal{U}} V_{I}$ and the result follows.

\section{REEB TyPE AND $K$-CONTACT}

In this section we prove a version of Proposition 2.1 of Yamazaki [Y] that relates torus actions and $K$-contactness. Recall that the Reeb vector field $R_{\alpha}$ of a contact form $\alpha$ on a manifold $B$ is the vector field uniquely defined by the equations

$$
\iota\left(R_{\alpha}\right) d \alpha=0, \quad \iota\left(R_{\alpha}\right) \alpha=1 .
$$

The Reeb vector field defines a splitting of the tangent bundle of $B$,

$$
T B=\xi \oplus \mathbb{R} R_{\alpha},
$$

where $\xi=\operatorname{ker} \alpha$ is the contact distribution. Since $\left(\xi,\left.d \alpha\right|_{\xi}\right)$ is a symplectic vector bundle, there exists a complex structure $J$ on $\xi$ compatible with $\left.d \alpha\right|_{\xi}$ so that $g_{\xi}=$ $\left.d \alpha\right|_{\xi}(\cdot, J \cdot)$ is a metric on $\xi$. Using (3.1) we may extend $g_{\xi}$ by zero to all of $T B$. Then $g=g_{\xi} \oplus \alpha \otimes \alpha$ is a Riemannian metric on $B$ in which $\xi$ and $R_{\alpha}$ are orthogonal and the length of the Reeb vector field is 1 . The metric $g$ is said to be adapted to the contact form $\alpha$. If additionally the Reeb vector field is Killing with respect to an adapted metric $g$, i.e., if the Lie derivative $L_{R_{\alpha}} g$ is zero, then the pair $(\alpha, g)$ is called a $K$-contact structure on $B$. If given a contact distribution $\xi$ on a manifold $B$ there exists a $K$-contact structure with $\operatorname{ker} \alpha=\xi$, we will say that $(B, \xi)$ admits a $K$-contact structure.

Note that if a Lie group $G$ acts on $B$ preserving a contact form $\alpha$, then it preserves the Reeb vector field $R_{\alpha}$, the contact distribution $\xi=\operatorname{ker} \alpha$ and the symplectic structure $\left.d \alpha\right|_{\xi}$. Therefore if $G$ is compact we may choose the complex structure $J$ (and hence the adapted metric $g$ ) to be $G$-invariant.

Proposition 3.1. A compact contact manifold $(B, \xi=\operatorname{ker} \alpha)$ admits the structure of a $K$-contact manifold if and only if there exists an action of a torus $G$ on $B$ preserving $\alpha$ and a vector $X \in \mathfrak{g}$ such that the function $\iota\left(X_{B}\right) \alpha=\left\langle\Psi_{\alpha}, X\right\rangle$ is strictly positive, i.e., the $G$ action is of Reeb type. Here as before $X_{B}$ denotes the vector field on $B$ induced by $X \in \mathfrak{g}$ and $\Psi_{\alpha}$ denotes the $\alpha$-moment map.

Proof. Suppose the action of a torus $G$ on $(B, \xi=\operatorname{ker} \alpha)$ is of Reeb type, i.e., suppose there is a vector $X \in \mathfrak{g}$ such that $\left\langle\Psi_{\alpha}, X\right\rangle$ is strictly positive (note that this is a condition on the co-oriented contact distribution $\xi$ and not just on the contact form $\alpha$ ). We then can multiply $\alpha$ by a positive $G$-invariant function $f$ so that $\left\langle\Psi_{f \alpha}, X\right\rangle=1$ ( take $f=1 /\left\langle\Psi_{\alpha}, X\right\rangle$ ). Therefore it is no loss of generality to assume that $\alpha\left(X_{B}\right)=\left\langle\Psi_{\alpha}, X\right\rangle=1$. Since the $G$ action preserves $\alpha$, we have $0=L_{X_{B}} \alpha=d \iota\left(X_{B}\right) \alpha+\iota\left(X_{B}\right) d \alpha=d 1+\iota\left(X_{B}\right) d \alpha$. Therefore $X_{B}$ is the Reeb vector field of $\alpha$. Now choose a $G$-invariant metric $g$ adapted to $\alpha$. Then, since $\alpha$ is $G$-invariant, $L_{X_{B}} g=0$, and so $(\alpha, g)$ is a $K$-contact structure on $(B, \xi)$.

Conversely suppose $(\alpha, g)$ is a $K$-contact structure on $B$. Since $B$ is compact, the group of isometries of $(B, g)$ is a compact Lie group $H$. Take the closure inside $H$ 
of the flow of the Reeb vector field $R_{\alpha}$. The closure is a compact connected abelian group $G$, i.e., a torus. Since the flow of $R_{\alpha}$ preserves the contact form $\alpha$, the action of $G$ preserves $\alpha$ as well. By construction $R_{\alpha}=X_{B}$ for some vector $X$ in the Lie algebra of $G$. Since $R_{\alpha}$ is a Reeb vector field we have $1=\iota\left(R_{\alpha}\right) \alpha=\left\langle\Psi_{\alpha}, X\right\rangle$, where $\Psi_{\alpha}: B \rightarrow \mathfrak{g}^{*}$ is the moment map for the action of $G$ on $(B, \alpha)$. Hence the action of $G$ on $(B, \xi=\operatorname{ker} \alpha)$ is of Reeb type.

We end the paper with an observation of the referee.

Proposition 3.2. Any (compact connected) contact toric manifold with an invariant $K$-contact structure is of Reeb type.

Proof. Suppose an effective action of a torus $G$ on a manifold $B$ is completely integrable (i.e., $\operatorname{dim} B+1=2 \operatorname{dim} G$ ) and preserves a $K$-contact structure $(\alpha, g)$. Let $\Psi_{\alpha}: B \rightarrow \mathfrak{g}^{*}$ denote the associated moment map. Then the group $G$ is a maximal torus in the Lie group of isometries of $(B, g)$. The Reeb vector field $R_{\alpha}$ of $\alpha$ is $G$-invariant, hence the torus action it generates commutes with $G$. But $G$ is a maximal torus, hence $R_{\alpha}$ is induced by a vector $X$ in the Lie algebra $\mathfrak{g}$ of $G$. It follows from the definitions that

$$
\left\langle\Psi_{\alpha}, X\right\rangle=\alpha\left(R_{\alpha}\right)=1>0,
$$

hence $(B, \operatorname{ker} \alpha)$ is of Reeb type.

\section{ACKNOWLEDGMENTS}

I thank Charles Boyer, Sue Tolman and Bill Graham for a number of useful conversations. I thank the referee for a number of very useful comments and in particular for Proposition 3.2 (the statement and the proof).

\section{REFERENCES}

[BG] C. P. Boyer and K. Galicki, A note on toric contact geometry, J. Geom. Phys. 35 (2000) 288-298; http://xxx.lanl.gov/abs/math.DG/9907043v2. MR 2001h:53124

[DJ] M.W. Davis and T. Januszkiewicz, Convex polytopes, Coxeter orbifolds and torus actions, Duke Math. J. 62 (1991), no. 2, 417-451. MR 92i:52012

[D] T. Delzant, Hamiltoniens périodiques et images convexes de l'application moment, Bull. Soc. Math. France 116 (1988), no. 3, 315-339. MR 90b:58069

[GS] V. Guillemin and S. Sternberg, Geometric quantization and multiplicities of group representations, Invent. Math. 67 (1982), 515-538. MR 83m:58040

[L] E. Lerman, Contact toric manifolds, http://xxx.lanl.gov/abs/math.SG/0107201

[LS] E. Lerman and N. Shirokova, Completely integrable torus actions on symplectic cones, Math. Res. Lett. 9 (2002). MR 2003e:53106

[S] R. Sjamaar, Holomorphic slices, symplectic reduction and multiplicities of representations, Ann. Math. 141 (1995), 87-129. MR 96a:58098

[TZ] J. Toth and S. Zelditch, Riemannian manifolds with uniformly bounded eigenfunctions, Duke Math Journal 111 (2002), 97-132. See also http://xxx.lanl.gov/abs/math-ph/0002038. MR 2003d:58048

[Y] T. Yamazaki, A construction of $K$-contact manifolds by a fiber join, Tohoku Math. J. 51 (1999), 433-446. MR 2001e:53094

Department of Mathematics, University of Illinois, Urbana, Illinois 61801

E-mail address: lerman@math.uiuc.edu 